\title{
Tachycardia induced tachycardia: case report of right ventricular outflow tract tachycardia and AV nodal reentrant tachycardia
}

\author{
M Cooklin, J M McComb
}

Regional

Cardiothoracic

Centre, Freeman

Hospital, High Heaton,

Newcastle-upon-Tyne

NE7 7DN, UK

M Cooklin

$\mathrm{J}$ M McComb

Correspondence to:

Dr Cooklin.

Accepted for publication 5 October 1998

\begin{abstract}
Tachycardia induced tachycardia, or so called double tachycardia, is rare. A 34 year old woman is described who had a history of syncope, frequent extrasystoles, and episodes of non-sustained ventricular tachycardia, perceived as palpitation, without syncope. At electrophysiological study, during infusion of isoprenaline, an episode of non-sustained ventricular tachycardia arising from the right ventricular outflow tract initiated sustained atrioventricular nodal reentrant tachycardia, thought to be the cause of the patient's syncope. Ablation of the right ventricular outflow tract focus abolished the ventricular ectopy; the slow AV nodal pathway was also ablated. The patient no longer has either syncope or palpitation.

(Heart 1999;81:321-322)
\end{abstract}

Keywords: syncope; double tachycardia; non-sustained ventricular tachycardia

Tachycardia induced tachycardia or "double tachycardias" are both rare. We report a case in which non-sustained ventricular tachycardia led to atrioventricular (AV) node reentrant tachycardia.

\section{Case report}

A 34 year old woman was referred for investigation of palpitations. She described two distinct types, the first severe, prolonged, and infrequent, and the second less severe and shorter, which occurred most days and was often related to exercise. There was no evidence of structural heart disease. Monitoring and exercise ECG revealed extrasystoles with a morphology consistent with a right ventricular outflow tract (RVOT) origin. She was

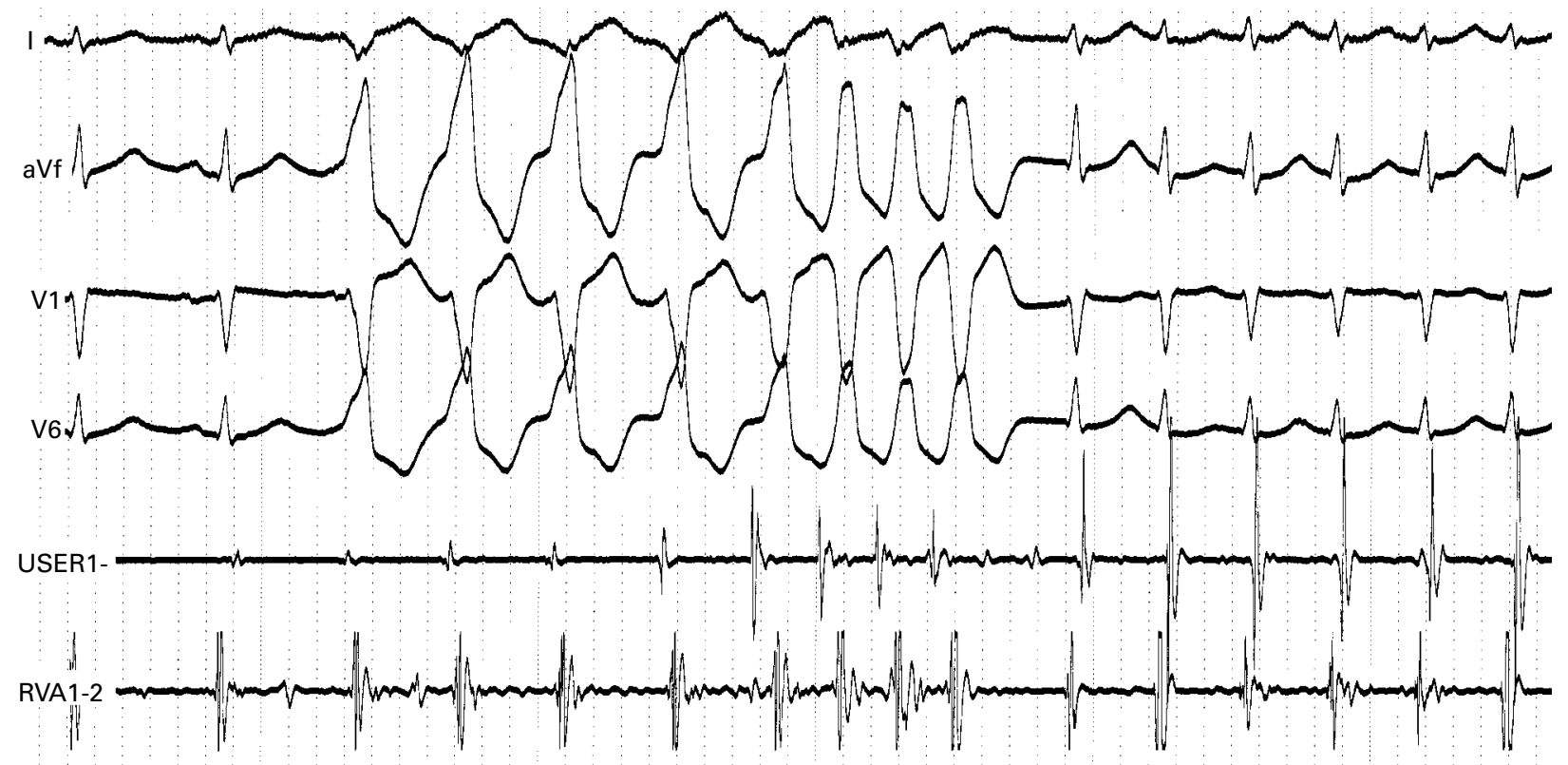

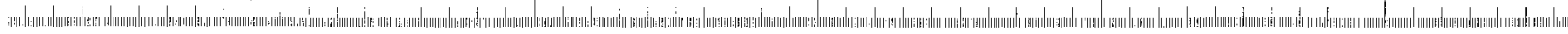

Figure 1 Initiation of supraventricular tachycardia by RVOT ectopy. Surface leads I, aVf, V1, V6, and electrograms recorded from the right ventricular apex (RVA 1-2) and RVOT (USER 1-) are shown. Following a short run of non-sustained ventricular tachycardia, narrow complex tachycardia is seen, cycle length $320 \mathrm{~ms}$. (Paper speed $50 \mathrm{~mm} / \mathrm{s}$.) 
treated with verapamil but admitted soon after with further palpitations and an episode of collapse.

An electrophysiological study was done in the postabsorptive state. Initially a quadripolar catheter was placed in the right ventricular apex and a Polaris ablation catheter to the RVOT for mapping and ablation. RVOT extrasystoles were observed only during isoprenaline infusion. On two occasions these led to a rapid narrow complex supraventricular tachycardia (fig 1). A further quadripolar catheter was advanced to the high right atrium that demonstrated no retrograde conduction during RVOT ectopy. Activation and pace mapping were used to guide ablation, and following three applications of radiofrequency energy to the RVOT no further ectopy was observed at baseline or during isoprenaline infusion.

A catheter was then positioned across the tricuspid valve to record the His deflection, and a diagnostic study performed. This revealed absent retrograde conduction during ventricular pacing, and dual AV nodal physiology with single AV node echo beats during atrial extrastimulus testing. Atrial pacing during isoprenaline induced AV node reentrant tachycardia. Four applications of radiofrequency energy were delivered to the posteroseptal region of the right atrium guided by electrogram morphology. Subsequently, no slow AV nodal pathway conduction could be demonstrated.

At review one month later she had returned to work and was experiencing no palpitations.

\section{Discussion}

In this patient, narrow complex tachycardia was twice induced by spontaneous nonsustained ventricular tachycardia originating in the RVOT. The only arrhythmia documented clinically had been ventricular ectopic beats arising from the RVOT; because the patient was extremely symptomatic, it was thought reasonable to ablate the origin of these. ${ }^{1}$ Atrioventricular nodal reentrant tachycardia (AVNRT) was subsequently induced by pacing, and the slow pathway ablated, although this had not been documented clinically (apart from the patient's description of two separate types of palpitations).

One tachycardia inducing another, with a completely separate mechanism, is uncommon. Double tachycardia, defined as the simultaneous occurrence of supraventricular and ventricular tachycardia (or atrial and junctional tachycardia) has been rarely reported, and usually occurs in patients with poor left ventricular function or in association with digoxin treatment. ${ }^{2-4}$
Eldar et al described three patients with exercise induced double tachycardia. ${ }^{5}$ In one patient, there was a background of ischaemic heart disease (atrial tachycardia and ventricular tachycardia). The other two patients had no evidence of structural heart disease, although one patient (with atrial flutter and polymorphic VT) had experienced recurrent episodes of aborted sudden death.

There do not appear to be any previous reports relating dual AV node physiology or AVNRT in patients with RVOT tachycardia or visa versa, and there is no reason to assume that it is anything more than a chance association. Induction of supraventricular tachycardia requires a substrate- for example, dual AV nodal pathways, and a trigger - for example, atrial or ventricular ectopic beats. In this patient, the absence of ventriculoatrial conduction, either during ventricular pacing or in response to RVOT ectopy, makes it difficult to explain the induction of AVNRT by the ventricular ectopy. It is possible that concealed retrograde penetration of the AV node occurred allowing the next antegrade impulse to depolarise the ventricle via the slow pathway and thus initiate tachycardia; no atrial signal was available and at onset of AVNRT, no P waves are visible. An alternative explanation is that spontaneous RVOT ectopy was dependent on catecholamine infusion, and this facilitated induction of AVNRT, possibly via atrial premature beats that were concealed by the ventricular ectopy.

RVOT tachycardia was not initiated by AVNRT in the this case. This is not surprising, as RVOT tachycardia is believed to be a triggered arrhythmia, whose induction would be favoured by high catecholamine states rather than rapid pacing. ${ }^{6}$

In conclusion we report a patient in whom one tachycardia led to the induction of a second. The second was still inducible following successful ablation of the first, but was then eliminated by radiofrequency ablation.

1 Gumbrielle T, Bourke JP, Furniss SS. Is ventricular ectopy a legitimate target for ablation? Br Heart $\mathcal{F} 1994 ; 72: 492-4$.

2 Castellanos A Jr, Azan L, Calvino JM. Simultaneous tachycardias. Am Heart $\mathcal{F}$ 1968;59:358-73.

3 Wishner SH, Kastor JA, Yurchak PM. Double atrial and atrioventricular junctional tachycardia. N Engl f Med 1972; 287:552-3.

4 Belhassen B, Pelleg A, Paredes A, et al. Simultaneous AV nodal reentrant and ventricular tachycardias. PACE 1984; 7:325-31

5 Eldar M, Belhassen B, Hod H, et al. Exercise-induced double (atrial and ventricular) tachycardia: a report of three cases. F Am Coll Cardiol 1989;14:1376-81.

6 Lerman BB, Belardinelli L, West GA, et al. Adenosinesensitive ventricular tachycardia: evidence suggesting cyclic AMP-mediated triggered activity. Circulation 1986;74:270 80 . 